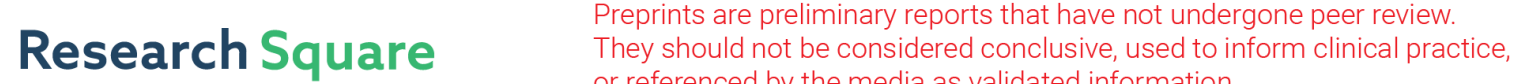 or referenced by the media as validated information. \\ Enhancement of Phenolics Content and Biological Activities of Longan (Dimocarpus Longan Lour.) Treated with Thermal and Aging Process
}

\section{Preaploy Hong-in}

Chiang Mai University

Waranya Neimkhum

Huachiew Chalermprakiet University

Chanun Punyoyai

Chiang Mai University

Suwannee Sriyab

Chiang Mai University

Wantida Chaiyana ( $\nabla$ Wantida.chaiyana@gmail.com )

Chiang Mai University

\section{Research Article}

Keywords: Dimocarpus longan, antioxidant, anti-hyaluronidase, gallic acid, corilagin, ellagic acid

Posted Date: April 7th, 2021

DOl: https://doi.org/10.21203/rs.3.rs-378198/v1

License: (c) (i) This work is licensed under a Creative Commons Attribution 4.0 International License.

Read Full License

Version of Record: A version of this preprint was published at Scientific Reports on August 5th, 2021. See the published version at https://doi.org/10.1038/s41598-021-95605-3. 


\section{Abstract}

This study is the first to successfully produce novel black Dimocarpus longan by undergoing thermal and ageing process. Pericarp, aril, and seed of black $D$. longan were macerated in $95 \% \mathrm{v} / \mathrm{v}$ ethanol. Their chemical compositions were investigated by Folin-Ciocalteu assay, aluminium chloride assay, and highperformance liquid chromatography. Antioxidant activities were evaluated in terms of radical scavenging and iron (III) reducing capacity. Enzyme inhibitory assay was used to evaluate the hyaluronidase inhibition. Black $D$. longan seed extract contained the significantly highest content of flavonoids and phenolic compounds $(p<0.05)$. Each gram of the extract contained $53.6 \pm 0.9 \mathrm{mg}$ of gallic acid, $19.8 \pm 2.9$ $\mathrm{mg}$ of corilagin, and $24.5 \pm 0.7 \mathrm{mg}$ of ellagic acid. This extract was the most potent anti-hyaluronidase and antioxidant with the strongest free radical scavenging activity and reducing power. Therefore, it was proposed as functional food and further used in the pharmaceutical or cosmeceutical industries.

\section{Introduction}

Dimocarpus longan Lour., a subtropical evergreen plant in the family of Sapindaceae, is widely known as longan. D. longan can be cultivated in several countries in East Asia, South-East Asia, Australia, and some subtropical regions in the US. ${ }^{1}$ China and Thailand are the largest area of commercial $D$. longan cultivation. $^{2}$ The succulent and edible aril with delicious flavor and health benefits lead $D$. longan to be more and more popular. ${ }^{1}$ Since the aril part of $D$. longan contains several polyphenols, flavonoids, organic acids, and polysaccharides, it possessed various beneficial biological activities, including antioxidant, antiglycation, anticancer, immunomodulatory, prebiotic, anti-osteoporotic, anxiolytic, and memoryenhancing effects. ${ }^{3}$ A decoction of the dried aril has been taken as a tonic for insomnia and neurasthenic neurosis treatment since an ancient time. ${ }^{4}$ Not only the aril part of $D$. longan, which is the only edible portion, that has been reported for the health-beneficial effects, the pericarp has also been reported to contain abundant polyphenols, flavonoids, and polysaccharides, which possessed antioxidant, antityrosinase, and anti-hyperglycaemic activities. ${ }^{1}$ On the other hand, $D$. longan seed, which is a waste from the food and canning industry, contains antioxidative polyphenols and possesses anti-tyrosinase, antibacterial, and anti-fungal activity. ${ }^{1,5,6,7}$ Besides, $D$. longan seed has been administered to counteract heavy sweating, whereas, the pulverized kernel was used for astyptic treatment according to the components of saponin, tannin, and fat ${ }^{8}$ Although $D$. longan have been reported to contain variety of biological active components and have a potential to be used for the treatment of various conditions, the fruit of $D$. longan has a short storage life since its pericarp rapidly turns brown and harden at ambient temperature. ${ }^{1,9}$ Even if low temperature $\left(1-5^{\circ} \mathrm{C}\right)$ could preserve $D$. longan from pathological decay, the fruit deteriorate easily after remove from the cold storage. ${ }^{3}$ Therefore, dried flesh and the canned product of $D$. longan are widely consumed and can be distributed worldwide. Generally, dried foods can be kept for a longer period, but their sensory and nutritional characteristics are often lost along with the water removal during the drying processes. ${ }^{10}$ Production of intermediate moisture food (IMF) is another technique to overwhelm this problem since the properties of IMF are close to fresh foods yet having 
extended the shelf life. ${ }^{10,11}$ A reduction of the moisture content and a water activity below 0.6 will not support the microbial growth and leads to shelf-stable products. ${ }^{11,12}$ Various types of food have been preserved as IMF, such as meat and several fruits, e.g. grapes, tomatoes, peaches, prunes, apricots, strawberries, etc. ${ }^{10,13}$ However, some additives are required in the production of IMF for antimicrobial (e.g. preservatives, sugar, and salt), along with the agents for water activity reducing and plasticizing, e.g. glycols and sorbitol. ${ }^{11,12}$ Beside IMF, the heating and ageing process could also prolong the shelf life without refrigeration. A well-known food undergone this process is black garlic (Allium sativum L.), a processed garlic produced by thermal treatment on raw garlic at high temperature and high relative humidity for 60-90 days without using additives. ${ }^{14,15}$ During the production process, raw garlic also undergoes Maillard reaction, which occurs between amine groups and carbonyl compounds, finally resulting in brownish melanoidin. ${ }^{16} \mathrm{~A}$ variety of biological activities of melanoidin has been demonstrated, including antioxidant, antibacterial, anti-inflammatory, hypoglycemic, hypotensive, antitumor effects, prevention of obesity, lowering of serum lipopolysaccharide levels, and modulation of the composition of the gut microbiota. ${ }^{16,17}$ Additionally, inhibition against oxidation and angiotensin I converting enzyme were enhanced in black garlic comparing to raw garlic. ${ }^{15}$ Therefore, the heating and ageing process not only preserved the food but also enhanced their biological activities. Since the production of black $D$. longan undergone heating and ageing process has not been reported before, this study is the first to produce a novel black $D$. longan and investigate the chemical compositions, as well as its health benefit potential in antioxidant, anti-inflammatory, and anti-hyaluronidase activities.

\section{Results And Discussion}

\section{DriedD. longanand blackD. longanextracts}

The external appreance of dried $D$. longan were totally different from black $D$. longan as shown in Fig. 1 . The color of pericarp, aril, and seed of black $D$. longan was obviously darker than that from dried $D$. longan, especially the aril part which turn from dark brownish color to black color. The pericarp and seed of black $D$. longan were substantially more moist than dried $D$. longan. The outer part of dried $D$. longan seed was shriveled, whereas, the dried $D$. longan pericarp was dry and brittle. All $D$. longan extracts were semisolid mass with different color as shown in Fig. 1. The color of black $D$. longan extracts were darker than the dried $D$. longan extracts. The color of pericarp extracts were the darkest, followed by the extract from seed and aril, respectively. Yields of each $D$. longan extracts are shown in Fig. 2. The aril part yielded the highest extract content, followed by pericarp and seed, respectively. The highest yield of the extract was obtained from black $D$. longan aril $(21.6 \% \mathrm{w} / \mathrm{w})$, followed by dried $D$. longan aril $(17.6 \% \mathrm{w} / \mathrm{w})$, dried $D$. longan pericarp $(13.8 \% \mathrm{w} / \mathrm{w})$, black $D$. longan aril $(11.0 \% \mathrm{w} / \mathrm{w})$, black $D$. longan seed $(6.6 \% \mathrm{w} / \mathrm{w})$, and dried $D$. longan seed $(3.6 \% \mathrm{w} / \mathrm{w})$, respectively.

\section{Chemical compositions of driedD. longanand blackD. longanextracts}

D. longan extracts were investigated for the content of total phenolic compounds, total flavonoid, gallic acid, corilagin, and ellagic acid. Gallic acid and corilagin are natural polyphenolic compounds which 
belong to hydrolyzable tannin, whereas, ellagic acid belongs to a flavonoid group. ${ }^{18}$ Among different parts of dried $D$. longan, pericarp extracts contained the significantly highest total phenolic content $(\mathrm{p}<$ 0.05 ) and the highest total flavonoid as shown in Fig. 3. The results were in a great agreement with the previous study reported that polyphenolic compounds are abundant in pericarp and seed of $D$. longan compared to the $D$. Iongan aril. ${ }^{1}$ The total phenolic content of pericarp, seed, and aril extracts from dried D. longan, which were $967.6 \pm 31.5,739.3 \pm 62.3$, and $229.5 \pm 2.6 \mu \mathrm{g}$ GAE per g extracts, were found in agreement with the previous study reported that the total phenolic content of $D$. longan were in the range of 22.09-132.47 mg of gallic acid equivalents (GAE/100 g), which were equivalent to 220.9-1,324.7 $\mu \mathrm{g}$ GAE per $g$ extracts. ${ }^{1}$ Interestingly, the dramatically enhanced in total phenolic content was observed in black $D$. longan seed extract. The ethanolic extract from black $D$. longan seed contained as high as $1827.1 \pm 73.1 \mu \mathrm{g}$ GAE per $g$ extracts, which was much higher that the previous reported. ${ }^{1}$ On the other hand, there was no significantly difference between the total phenolic content of dried and black $D$. longan extract from pericarp and aril $(p>0.05)$.

In addition to the total phenolic content, black $D$. longan seed extract also contained the significantly highest flavonoids content $(\mathrm{p}<0.05)$. Among various dried $D$. longan extracts, the pericarp part contained the significantly highest flavonoid content of $2.8 \pm 2.4 \mu$ g QE per g extract as shown in Fig. $3(p<0.05)$. The results were in a great accordance with the previous study reported that the quercetin content of $D$. longan pericarp were $3.12 \pm 0.76 \mathrm{mg} / \mathrm{kg}$, which was equivalent to $3.12 \pm 0.76 \mu$ g per g extract. ${ }^{19}$ Besides, $D$. longan pericarp has been reported to contain slightly higher content of flavonoids than $D$. longan seed and aril. ${ }^{20}$ Obviously, the flavonoid content of black $D$. longan seed extract, which was as high as $13.6 \pm$ $2.5 \mu \mathrm{g}$ QE per g extract, was dramatically enhanced, which was about four times higher than previously reported.

Although the thermal and ageing process of $D$. longan did not affect the phenolic and flavonoid content of $D$. longan pericarp and aril, the total phenolic and flavonoid content of $D$. longan seed were obviously enhanced after the production process of black $D$. longan. The likely explanations might be due to the formation of biological compounds, which were not originally present in the $D$. longan seed, during the thermal and ageing process. ${ }^{21}$

Gallic acid, corilagin, and ellagic acid have been identified as major polyphenolic components of $D$. longan pericarp and seed in several previous studies. $22,23,24$ Therefore, the content of these polyphenolic components were also investigated in black $D$. longan extract, in a comparison with dried $D$. longan extracts. The HPLC chromatograms of gallic acid, corilagin, and ellagic acid are shown in Fig. 4. The peak of gallic acid, corilagin, and ellagic acid were detected at around 3.7, 9.9, and $19.2 \mathrm{~min}$, respectively. The content of gallic acid, corilagin, and ellagic acid of each $D$. longan extracts are shown in Fig. 5 . The results were in a good agreement with their total phenolic and total flavonoid content since black $D$. longan seed extract contained the significantly highest polyphenolic compounds and flavonoids content of $(p<0.05)$. 
Among various parts of dried $D$. longan fruit, seeds contained the significantly highest content of gallic acid, corilagin, and ellagic acid $(p<0.05)$ with the amount of $5.3 \pm 0.0,8.9 \pm 0.1$ and $1.9 \pm 0.2 \mathrm{mg} / \mathrm{g}$ extract, respectively. Interestingly, the content of these phenolic and flavonoid were significantly enhnaced after the production process of black $D$. longan $(\mathrm{p}<0.05)$. The gallic acid, corilagin, and ellagic acid content of black $D$. longan seed extract were as high as $53.6 \pm 0.9,19.8 \pm 2.9$, and $24.5 \pm 0.7 \mathrm{mg} / \mathrm{g}$ extract, respectively. The content of gallic acid and ellagic acid were about ten times increased, whereas, the content of corilagin was double when the $D$. longan undergone thermal and ageing process. The reason might be due to the liberation of free polyphenolic and flavonoid from the bound forms (i.e. esterified and glycosylate) or the decline in enzymatic oxidation involving in the antioxidant compounds in the raw fruit. ${ }^{25}$ The results of black $D$. longan were in accordance with the black garlic as the total phenolic and total flavonoid content of the garlic subjected to the thermal processing steps were significantly higher than those of fresh garlic. ${ }^{21,25}$ The previous study reported that the phenolic content was increased by about 4-10-fold in the black garlic cloves compared with the fresh garlic. ${ }^{21}$

\section{Antioxidant activities of driedD. longanand blackD. longanextracts}

The antioxidant activities of dried and black $D$. longan extracts were investigated by two assays with different mechanisms of action. The ABTS assays referred to the electron transfer reaction and represented the radical scavenging activity of the tested samples, while the FRAP assay referred to the ion reduction process, which represented the ability of the tested compound to convert ferric ions $\left(\mathrm{Fe}^{+}\right)$ to ferrous ions $\left(\mathrm{Fe}^{+}\right) .{ }^{26,27,28}$ The ferric reducing antioxidant power $\left(\mathrm{EC}_{1}\right)$ and TEAC value of dried and black $D$. longan extracts are shown in Fig. 6.

The TEAC value of black $D$. longan extracts were not significantly different from dried $D$. longan extracts, except in the aril part. The dried $D$. longan aril extract had no antioxidant activity, whereas, the black $D$. longan aril extract possessed some antioxidant activity with the TEAC value of $4.1 \pm 1.4 \mu \mathrm{g}$ Trolox $/ \mathrm{mg}$ extract. The probable explanation lies in the greater Maillard reaction which occurs in the aril part as compared with the others. As Maillard reactions refer to a chemical reaction between an amino acid and a reducing sugar in the presence of heat ${ }^{29}, D$. longan aril, which contained glucose, fructose, and various types of amino acids, e.g. $Y$-aminobutyric acid, tended to undergone Maillard reaction. ${ }^{2}$ Besides, this nonenzymatic browning reactions led black $D$. longan a darker color and resulting in the formation of some antioxidant compounds. ${ }^{25}$

On the other hand, black $D$. longan pericarp and seed extracts possessed the same radical scavenging activity as that from dried $D$. longan. The likely possibility might be because of the degradation of some oxidative compounds during the heating process although some free polyphenolic and flavonoid were liberated from the bound forms. ${ }^{21}$ Interestingly, the TEAC values of pericarp and seed extracts from both dried and black $D$. longan were comparable to ascorbic acid, gallic acid, and corilagin ( $p>0.05)$. Ellagic acid was remarked as the most potent radical scavenger (TEAC $=23.4 \pm 0.3 \mu \mathrm{g}$ Trolox $/ \mathrm{mg}$ ), followed by ascorbic acid (TEAC $=12.3 \pm 0.0 \mu \mathrm{g}$ Trolox $/ \mathrm{mg})$, gallic acid $(\mathrm{TEAC}=12.8 \pm 0.2 \mu \mathrm{g}$ Trolox $/ \mathrm{mg})$, and 
corilagin (TEAC $=12.7 \pm 0.1 \mu \mathrm{g}$ Trolox $/ \mathrm{mg}$ ). Thereby, ellagic acid was found to be the main compound responsible for the free radical scavenging activity of $D$. longan extracts together with gallic acid and corilagin. ${ }^{30}$ Although the previous study reported that among various polyphenolic compounds, tannins demonstrated the strongest ABTS + radical scavenging activity ${ }^{31}$, the present study remarked that ellagic acid, which belongs to a flavonoid group, was more potent than gallic acid and corilagin, which belong to hydrolyzable tannin. ${ }^{1}$ Furthermore, $D$. longan extracts from both pericarp and seed part could be hence remarked as the natural extracts with potent radical scavenging activity.

Aside from radical scavenging activity, $D$. longan extracts also possessed a reducing ability as shown in Fig. 6 . The reducing ability of $D$. longan extracts were in a very well accordance with their phenolic and flavonoid content. Gallic acid possessed the significantly highest $\mathrm{EC}_{1}$ value of $237.0 \pm 1.6 \mathrm{mM} \mathrm{FeSO} \mathrm{m}_{4} / \mathrm{mg}_{\text {, }}$ which was comparable to that of ascorbic acid $\left(238.3 \pm 0.2 \mathrm{mM} \mathrm{FeSO}_{4} / \mathrm{mg}\right)$, followed by corilagin (226.2 $\left.\pm 2.9 \mathrm{mM} \mathrm{FeSO}_{4} / \mathrm{mg}\right)$ and ellagic acid $\left(192.3 \pm 0.7 \mathrm{mM} \mathrm{FeSO}_{4} / \mathrm{mg}\right)$, respectively. However, both phenolic and flavonoid were responsible for their reducing capacity. ${ }^{28}$ The black $D$. longan seed extract, which contained the highest content of total phenolic, total flavonoid, gallic acid, corilagin, and ellagic acid, thus possessed the significantly highest reducing ability with the $\mathrm{EC}_{1}$ value of $150.0 \pm 1.0 \mathrm{mM} \mathrm{FeSO}_{4} / \mathrm{mg}$ extract $(p<0.05)$. Consequently, the black $D$. longan seed extract was suggested as the most potent antioxidant extract with the strongest free radical scavenging and reducing ability.

\section{Anti-inflammatory activities of driedD. longanand blackD. longanextracts}

The inhibitory activities against the secretion of IL- 6 and TNF- $a$, which are key players involved in the agerelated inflammatory process ${ }^{32}$, of dried and black $D$. longan extracts were investigated and the results are shown in Fig. 7. TNF-a is known as an indicator of chronic inflammatory processes related to ageing, whereas, IL- 6 has been noted as the cytokine for geriatricians, which are associated with poor physical performance, muscle weakness, and could predict the onset of disability. ${ }^{33,34}$ Among various parts of $D$. longan fruit, aril of both dried and black $D$. longan were predominant in the IL- 6 and TNF-a inhibition. Gallic acid was suggested to be the main compound responsible for both IL- 6 and TNF-a inhibitory activities. In contrast, corilagin was responsible only for TNF-a inhibition. Although $D$. longan extracts and their major chemical components exhibited only low to moderate anti-inflammatory activities comparing to dexamethasone, a corticosteroid used in the treatment of inflammations, they were suggested as natural anti-inflammatory products with no steroidal side effect.

\section{Anti-hyaluronidase activities of driedD. longanand blackD. longanextracts}

Hyaluronidase, a homologous enzyme that hydrolysis or depolymerise hyaluronan, plays an important role in the modulating activity of many pathological processes. ${ }^{35}$ Hyaluronan plays a pivotal role in the maintenance of the elastoviscosity of liquid connective tissues and control the water transportation that was related to the tissue hydration. ${ }^{36}$ The degradation of hyaluronan resulting in the production of breakdown products, which are the signal that injury has occurred and initiates an inflammatory 
response. ${ }^{35}$ Additionally, hyaluronan has been known an lubricant and shock-absorber in joints and connective tissues. ${ }^{37}$ Its degradation hence leads to the deterioration of viscoelastic properties of the synovial fluid. ${ }^{38}$

The inhibitory activities against hyaluronidase of dried and black $D$. longan extracts are shown in Fig. 8 . Although $D$. longan extracts exhibited low anti-hyaluronidase actvitiy, the inhibitory effect of black $D$. longan seed was significantly enhanced compared to the dried $D$. longan seed extract. Since the antihyaluronidase activity of black $D$. longan seed extract $(18.4 \pm 2.0 \%)$ was the most significantly potent $(p<$ $0.05)$, black $D$. longan seed extract could be suggested for the anti-hyaluronidase apart from its antioxidant activities.

In conclusion, Black $D$. longan was successfully produced after undergone thermal and ageing process in the controlled temperature of $70^{\circ} \mathrm{C}$ and controlled humidity of $75 \% \mathrm{RH}$. The external appearance of black $D$. longan was totally different from that of dried $D$. longan. The ethanolic extract of black $D$. longan seed contained the most significantly abundant of total phenolic, total flavonoid, gallic acid, corilagin, and ellagic acid content $(p<0.05)$. Furthermore, the black $D$. longan seed extract possessed the most significantly potent antioxidant and anti-hyaluronidase activities. The radical scavenging activity, reducing power, and anti-hyaluronidase activities of black $D$. longan seed extract were obviously higher than that of dried $D$. longan seed extract and the previously reports. Therefore, black $D$. longan seed extract was suggested for further used topically for the promotion of various health benefits, such as antiskin ageing, joint pain reliever, etc. On the other hand, the aril part of both dried and black $D$. longan possessed significant anti-inflammatory activities. They were suggested as natural edible antiinflammatory agents.

\section{Material And Methods}

\section{Chemical material}

L-Ascorbic acid, aluminium chloride ( $\mathrm{AlCl}_{3}$ ), 2,2'-azino-bis3-ethylbenzothiazoline-6-sulfonic acid (ABTS), calcium chloride $\left(\mathrm{CaCl}_{2}\right)$, corilagin, dexamethasone, disodium phosphate $\left(\mathrm{Na}_{2} \mathrm{HPO}_{4}\right)$, ferric chloride $\left(\mathrm{FeCl}_{3}\right)$, ferrous chloride $\left(\mathrm{FeCl}_{2}\right)$, ferrous sulfate $\left(\mathrm{FeSO}_{4}\right)$, formic acid, Folin-ciocalteu reagent, 4,4',5,5',6,6'hexahydroxy-diphenic acid 2,6,2',6'-dilactone (ellagic acid), hyaluronic acid, hydrochloric acid ( $\mathrm{HCl}), 6$ hydroxy-2,5,7,8-tetramethylchroman-2-carboxylic acid (Trolox), bovine testicular hyaluronidase (E.C.3.2.1.3.5), potassium acetate $\left(\mathrm{CH}_{3} \mathrm{COOK}\right)$, potassium persulphate $\left(\mathrm{K}_{2} \mathrm{~S}_{2} \mathrm{O}_{8}\right)$, sodium acetate $\left(\mathrm{C}_{2} \mathrm{H}_{3} \mathrm{NaO}_{2}\right)$, sodium carbonate $\left(\mathrm{Na}_{2} \mathrm{CO}_{3}\right)$, sodium chloride $(\mathrm{NaCl})$, sodium dihydrogen phosphate $\left(\mathrm{NaH}_{2} \mathrm{PO}_{4}\right)$, sodium phosphate $\left(\mathrm{Na}_{3} \mathrm{PO}_{4}\right), 2,4,6$-tripyridyl-striazine (TPTZ), and 3,4,5-trihydroxybenzoic acid (gallic acid) were analytical grade bought from Sigma-Aldrich (St. Louis, MO, USA). Amphotericin B, Dulbecco modified eagle medium (DMEM), L-glutamine, penicillin/streptomycin, trypan blue, and secondary antibody conjugated with HRP were bought from Invitrogen ${ }^{\text {TM }}$ (Carlsbad, CA, USA). Lipopolysaccharide (LPS) was bought from Cell Signaling Technology ${ }^{\circledR}$ (Danvers, MA, USA). GlutaMAX ${ }^{\mathrm{TM}}$ - 
I supplement was bought from Thermo Fisher Scientific, Inc. (ThermoFisher Scientific, Waltham, MA, USA). Newborn bovine calf serum, fetal bovine serum (FBS), bovine serum albumin (BSA), and antibioticantimycotic solution $(100 \times)$ was bought from Gibco $^{\text {TM }}$ (Thermo Fisher Scientific, Waltham, MA, USA). Acetic acid, ethanol, and dimethyl sulfoxide (DMSO), were purchased in analytical grade from Labscan, Ltd. (Dublin, Ireland). Acetonitrile was purchased in HPLC-grade from Labscan, Ltd. (Dublin, Ireland).

\section{Plant Material}

The D. longan fruits were collected from Chiang Mai Province in the Northern Thailand during 2020 by the gardeners according to WHO Guidelines on Good Agricultural and Collection Practices (GACP) for Medicinal Plants. ${ }^{39}$ The preparation of conventional dried and black $D$. longan was performed by Faculty of Agro-Industry, Chiang Mai University, Chiang Mai, Thailand.

\section{Conventional dried $D$. longan preparation}

Conventional dried $D$. longan was obtained after the whole fruit of fresh $D$. longan was incubated in an oven set the temperature of $50^{\circ} \mathrm{C}$ until dryness. The sample of dried $D$. longan was kept in sealed plastic bag to prevent contact with air and humidity in the room temperature until further experiments.

\section{Black $D$. longan preparation by thermal and aging process}

Black $D$. longan was obtained after the whole fruit of dried $D$. longan was incubated for 20 days in a controlled temperature of $70^{\circ} \mathrm{C}$ and controlled humidity of $75 \%$ relative humidity. The sample of black $D$. longan was kept in sealed plastic bag to prevent contact with air and humidity in the room temperature until further experiments.

\section{Preparation of dried $D$. longan and black $D$. longan extracts}

Seed, aril, and pericarp were separated from each other. Each part of $D$. longan fruit was ground into fine powder using 20-inch herbal medicine grinder tub with a powerful motor (Thai Pradith Industry Co., Ltd., Bangkok, Thailand). Dried $D$. longan powder was subsequently macerated in $95 \% \mathrm{v} / \mathrm{v}$ ethanol for 3 cycles of $24 \mathrm{~h}$. The extracting solvent from 3 cycles were combined and removed using a rotary evaporator (Buchi Labortechnik GmbH, Essen, Germany). All extracts were stored in the refrigerator $\left(4^{\circ} \mathrm{C}\right)$ until further experiments.

\section{Chemical compositions determination of dried $D$. longan and black $D$. longan extracts}

\section{Total phenolic content determination}

Each $D$. longan extract was analyzed for total phenolic content using the Folin-Ciocalteu method according to the previously described method. ${ }^{40}$ The results were presented in the form of gallic acid equivalent values (GAE) representing an amount of gallic acid $(\mu \mathrm{g})$ per $\mathrm{g}$ of the $D$. longan extracts. GAE was calculated following the equation; $X=(Y-0.0075) / 0.3812$, where $X$ is $G A E$ or $\mu g$ of gallic acid per $g$ 
of the $D$. longan extracts and $\mathrm{Y}$ is an absorbance of each sample tested in Folin-Ciocalteu assay. The experiments were triplicately performed.

\section{Total flavonoid content determination}

Total flavonoid content of each $D$. longan extracts was investigated using aluminium chloride method which has been previously described with some modifications. ${ }^{41}$ Firstly, $20 \mu \mathrm{l}$ of the ethanolic solution of D. longan extracts was mixed with $80 \mu \mathrm{l}$ of $10 \% \mathrm{w} / \mathrm{v} \mathrm{AlCl}$ a aueous solution and $100 \mu \mathrm{l}$ of $1 \mathrm{M} \mathrm{CH}_{3} \mathrm{COOK}$ aqueous solution. After the resulting mixtures were incubated for $30 \mathrm{~min}$ in the dark, they were measured for an absorbance at $415 \mathrm{~nm}$ using multimode detector (SPECTROstar Nano, BMG Labtech, Offenburg, Germany). Quercetin was applied as a standard compound to construct a calibration curve. Finally, the results were presented as quercetin equivalent (QE) values which represented a $\mu \mathrm{g}$ of quercetin per $\mathrm{g}$ of the $D$. longan extracts. QE was calculated following the equation; $X=(Y+0.033) / 0.107$, where $X$ is $Q E$ or $\mu \mathrm{g}$ of quercetin per $\mathrm{g}$ of the $D$. longan extracts and $\mathrm{Y}$ is an absorbance of each sample tested in aluminium chloride assay. The experiments were triplicately performed.

\section{Determination of gallic acid, corilagin, and ellagic acid content by high performance liquid chromatography (HPLC)}

The quantitative analysis of gallic acid, corilagin, and ellagic acid were performed using an HP 1100 chromatographic system (Hewlett-Packard, Waldbronn, Germany). A gradient mobile phase system composed of two phases, including phase $A(0.05 \%$ formic acid in acetonitrile) and phase $B(0.05 \%$ formic acid aqueous solution). The program was set for gradient elution of $10 \% \mathrm{~A}(0-8 \mathrm{~min}), 20 \% \mathrm{~A}$ (8$28 \mathrm{~min}), 30 \% \mathrm{~A}(28-30 \mathrm{~min})$, and $10 \% 10$ (30-35 min), eluting the sample at a flow rate of $1.0 \mathrm{ml} / \mathrm{min}$. The UV detector was set at $280 \mathrm{~nm}$ with a Eurospher II 100-5 C18 (250 ' 4.6 mm, i.d. $5 \mu \mathrm{m}$, Knauer, Berlin, Germany). All samples, standard solution, and mobile phase had been filtrated through a $0.45 \mathrm{~mm}$ millipore filter, type GV (Millipore, Bedford, MA) before injection to the HPLC system. The injected volume was set at $20 \mathrm{~mL}$. The sample of $D$. longan extracts was prepared at the concentration of $1 \mathrm{mg} / \mathrm{ml}$. Various concentrations of standard gallic acid $(10-150 \mu \mathrm{g} / \mathrm{ml})$, ellagic acid $(5-100 \mu \mathrm{g} / \mathrm{ml})$, and corilagin $(2-80 \mu \mathrm{g} / \mathrm{ml})$ solution were used for the construction of standard curves for quantitative determination. Subsequently, the content of gallic acid, corilagin, and ellagic acid was then calculated.

Gallic acid content was calculated following the equation; $X 1=(100 \mathrm{~A}+1,296) / 26.8 \mathrm{C}(\mathrm{R} 2=0.9964)$, where $X 1$ is the gallic acid concentration, $A$ is the area under the curve (AUC) of the gallic acid peak detected around $4 \mathrm{~min}$, and $\mathrm{C}$ is the concentration of the respective sample solution.

Corilagin content was calculated following the equation; $X 2=(100 \mathrm{~A}+2,325) / 17.8 \mathrm{C}(\mathrm{R} 2=0.9996)$, where $X 2$ is the concentration of corilagin, $A$ is the AUC of the corilagin peak detected around $10 \mathrm{~min}$, and $\mathrm{C}$ is the concentration of the respective sample solution.

Ellagic acid was calculated following the equation; $X 3=(100 \mathrm{~A}+13,372) / 33.7 \mathrm{C}(\mathrm{R} 2=0.9957)$, where $X 3$ is the concentration of ellagic acid, A is the AUC of the ellagic acid peak detected around $20 \mathrm{~min}$, and $\mathrm{C}$ is 
the concentration of the respective sample solution.

\section{Antioxidant activities determination of dried $D$. longan and black $D$. longan extracts}

\section{2, 2'-azinobis (3-ethylbenzothiazoline-6-sulfonic acid) (ABTS) assay}

The radical scavenging effects against $\mathrm{ABTS}^{*+}$ of $D$. longan extracts, gallic acid, corilagin, and ellagic acid were evaluated by ABTS assay and reported in the term of Trolox equivalent antioxidant capacity (TEAC) which represented the quantity of Trolox that equivalent to $1 \mathrm{mg}$ of the $D$. longan extracts. ${ }^{42}$ TEAC values were calculated following the equation; $X=(Y-1.2028) / 7.9964$, where $X$ is TEAC value and $Y$ is an absorbance of each sample tested in ABTS assay. L-Ascorbic acid was used as a positive control. The experiments were triplicately performed.

\section{Ferric reducing/antioxidant power (FRAP) assay}

The reducing capacity of $D$. longan extracts, gallic acid, corilagin, and ellagic acid were investigated by means of ferric ion reducing activities. ${ }^{40}$ The ferric reducing/antioxidant power of each $D$. longan extracts were expressed in the form of equivalent concentrations $\left(\mathrm{EC}_{1}\right)$ representing the amount of ferric-TPTZ reducing capacity, which is equivalent to $1 \mathrm{mg}$ of the $D$. longan extract. $\mathrm{EC}_{1}$ values were calculated following the equation; $X=(Y-0.0287) / 0.01405$, where $X$ is $E C_{1}$ value and $Y$ is an absorbance of each sample tested in FRAP assay. L-Ascorbic acid was used as a positive control. The experiments were triplicately performed.

\section{Anti-inflammatory activities determination of dried $D$. longan and black $D$. longan extracts}

Murine monocyte-macrophage (RAW 264.7) cells (American Type Culture Collection, ATCCTIB-71) treated with LPS were used to investigate the effect of $D$. longan extracts and their chemical compositions on the pro-inflammatory cytokine secretion (IL-6 and TNF-). The cell culture was performed according to a method previously described with some modifications. ${ }^{43,44}$ The treated cells along with its supernatant were divided into two parts. The first withdrawal part was undergone centrifugation for 10 min at 13,500' $\mathrm{g}$ and the supernatant was investigated for the cytokine secretion by enzyme-linked immunosorbent assay (ELISA) following the manufacturer's protocol (R\&D Systems). On the other hand, the second part, which was leftover in the wells, was investigated for the cell viability using 3-(4,5-dimethylthiazol-2-yl)-2,5diphenyltetrazolium bromide (MTT) assay. To reduce variation due to cell density differences, secretion of IL-6 and TNF-a from RAW 264.7 cells were normalized to MTT levels. ${ }^{44}$ RAW 264.7 cells without LPS treatment served as a negative control, while $100 \%$ of cytokine secretion was from positive control of which RAW 264.7 cells treated with LPS. The inhibitory activities of each samples were was calculated following the equation; \%inhibition $=100-A$, where $A$ is the amount of cytokines secretion.

Dexamethasone was served as a positive control for both IL-6 and TNF-a secretory inhibition. The experiments were triplicately performed.

\section{Anti-hyaluronidase activity determination of dried $D$. longan and black $D$. longan extracts}


The hyaluronidase inhibitory activity of $D$. longan extracts, gallic acid, corilagin, and ellagic acid was evaluated by measuring a product from the cleavage of sodium hyaluronate by hyaluronidase. ${ }^{45}$ Prior to the experiment, the enzyme activity of hyaluronidase was determined. Only more than $90 \%$ enzyme activity was used in the anti-hyaluronidase activity determination. The hyaluronidase inhibitory activity were calculated according to the following equation; \% Inhibition $=[1-X / Y)]^{\prime} 100$, where $X$ is the absorbance of the mixtures with sample; $Y$ is the absorbance of the mixtures without sample. Oleanolic acid was used as a positive control. The experiments were triplicately performed.

\section{Statistical analysis}

All values were given as means \pm standard and analyzed. The statistical analysis was used t-test and ANOVA by using SPSS software (SPSS Statistics 21.0, IBM Corporations, New York, NY, USA). A value of $p<0.05$ were accepted as significant.

\section{Declarations}

\section{Acknowledgements}

This research was funded by Agricultural Research Development Agency (ARDA), grant number PRP6305032210. The APC was funded by Research Center of Pharmaceutical Nanotechnology, Faculty of Pharmacy, Chiang Mai University and Innovation Center for Holistic Health, Nutraceuticals, and Cosmeceuticals, Faculty of Pharmacy, Chiang Mai University. Sriyab is grateful to CMU Presidential Scholarship 2020 for Post-Doctoral researcher.

\section{Author contributions statement}

W.C. conceived and designated the experiments. P.H. conducted the experiments. P.H., C.P., W.N., and S.S. collected and analyzed the data. P.H. and W.C. wrote the initial manuscript and revised the manuscript.

\section{References}

1. Zhang, X., Guo, S., Ho, C. T. \& Bai, N. Phytochemical Constituents and Biological Activities of Longan (Dimocarpus longan Lour.) Fruit: A Review. Food Sci. Hum. Well. 9 (2), 95-102 https://doi.org/10.1016/j.fshw.2020.03.001 (2020).

2. Yang, B., Jiang, Y., Shi, J., Chen, F. \& Ashraf, M. Extraction and pharmacological properties of bioactive compounds from longan (Dimocarpus longan Lour.) fruit-A review. Food Res. Inter. 44 (7), 1837-1842 https://doi.org/10.1016/j.foodres.2010.10.019 (2011).

3. Tang, Y. Y. et al. Polyphenols and alkaloids in byproducts of Longan fruits (Dimocarpus Longan Lour.) and their bioactivities. Molecules. 24 (6), 1186 (2019). https://doi.org/ 10.3390/ molecules 24061186 
4. Prasad, K., Neha, P., Lal, M. K. \& Gaurav, A. K. Longan (Dimocarpus longan Lour) processing: A Review. Int. J. Curr. Microbiol. Appl. Sci. 6 (8), 1-7 (2017).

5. Soong, Y. Y. \& Barlow, P. J. Quantification of gallic acid and ellagic acid from longan (Dimocarpus longan Lour.) seed and mango (Mangifera indica L.) kernel and their effects on antioxidant activity. Food Chem. 97 (3), 524-530 https://doi.org/10.1016/j.foodchem.2005.05 (2006).

6. Rangkadilok, N. et al. In vitro antifungal activities of longan (Dimocarpus longan Lour.) seed extract. Fitoterapia. 83 (3), 545-553 https://doi.org/10.1016/j.fitote.2011.12.023 (2012).

7. Tseng, H. C., Wu, W. T., Huang, H. S. \& Wu, M. C. Antimicrobial activities of various fractions of longan (Dimocarpus longan Lour. Fen Ke) seed extract. Int. J. Food Sci. Nutr. 65 (5), 589-593 https://doi.org/10.3109/09637486.2014.886181 (2014).

8. Ashok, A. D. \& Ravivarman, J. Kayalvizhi \& K. Nutraceutical Values of Minor Fruits on Immunity Development to Combat Diseases. Int. J. Curr. Microbiol. App. Sci. 9 (6), 1303-1311 https://doi.org/10.20546/ijcmas.2020.906.162 (2020).

9. Jiang, Y., Zhang, Z., Joyce, D. C. \& Ketsa, S. Postharvest biology and handling of longan fruit (Dimocarpus longan Lour.). Postharvest Biol. Technol. 26 (3), 241-252 https://doi.org/10.1016/S0925-5214(02)00047-9 (2002).

10. Qiu, L., Zhang, M., Tang, J., Adhikari, B. \& Cao, P. Innovative technologies for producing and preserving intermediate moisture foods: A review. Food Res. Int. 116, 90-102 https://doi.org/10.1016/j.foodres.2018.12.055 (2019).

11. Taoukis, P. S. \& Richardson, M. Principles of intermediate-moisture foods and related technology. Water activity in foods: fundamentals and applications.385-424. https://doi.org/10.1002/9781118765982.ch16 (2020).

12. Saha, S. I. M. F. To Enhance the Shelf-Life of Food. Technology. 6 (10), 103-108 https://doi.org/10.46501/IJMTST06108 (2020).

13. Rahim, M. A. et al. Present status of quality management in postharvest system for fruit in Bangladesh. In Europe-Asia Symposium on Quality Management in Postharvest Systems-Eurasia 2007. 804, 623-630. https://doi.org/10.17660/ActaHortic.2008.804.91 (2007).

14. Wu, J., Jin, Y. \& Zhang, M. Evaluation on the physicochemical and digestive properties of melanoidin from black garlic and their antioxidant activities in vitro. Food Chem. 340, 127934 https://doi.org/10.1016/j.foodchem.2020.127934 (2021).

15. Jing, H. \& Black Garlic Processing, Composition Change, and Bioactivity. eFood. 1(3), 242-246. https://doi.org/10.2991/efood. k.200617.001 (2020).

16. Yu, J., Shan, Y., Li, S. \& Zhang, L. Potential contribution of Amadori compounds to antioxidant and angiotensin I converting enzyme inhibitory activities of raw and black garlic. LWT. 109553. https://doi.org/10.1016/j.Iwt.2020.109553 (2020).

17. Wu, J. et al. Black garlic melanoidins prevent obesity, reduce serum LPS levels and modulate the gut microbiota composition in high-fat diet-induced obese C57BL/6J mice. Food Funct. 11 (11), 95859598 https://doi.org/10.1039/D0FO02379E (2020). 
18. Zeng, M. et al. Gallic Acid Inhibits Bladder Cancer T24 Cell Progression Through Mitochondrial Dysfunction and PI3K/Akt/NF-KB Signaling Suppression. Front. Pharmacol. 11, 1222 Front. Pharmacol. fphar.2020.01222 (2020).

19. Bai, X., Pan, R., Li, M., Li, X. \& Zhang, H. HPLC profile of Longan (cv. Shixia) pericarp-sourced phenolics and their antioxidant and cytotoxic effects. Molecules. 24 (3), 619 https://doi.org/10.3390/molecules24030619 (2019).

20. Liu, C. et al. Antioxidant capacity and contents of polyphenols in pericarps and stones of Dimocarpus longan. Food Sci. Technol. 39, 203-211 https://doi.org/10.3390/molecules24061186 (2014).

21. Kim, J. S., Kang, O. J. \& Gweon, O. C. Comparison of phenolic acids and flavonoids in black garlic at different thermal processing steps. J. Funct. Foods. 5 (1), 80-86 (2013). https://doi.org/ 10.1016/j.jff.2012.08.006

22. Zheng, G. et al. Polyphenols from longan seeds and their radical-scavenging activity. Food Chem. 116 (2), 433-436 https://doi.org/10.1016/j.foodchem.2009.02.059 (2009).

23. Prasad, K. N. et al. Enhanced antioxidant and antityrosinase activities of longan fruit pericarp by ultra-high-pressure-assisted extraction. J. Pharm. Biomed. Anal. 51 (2), 471-477 https://doi.org/10.1016/j.jpba.2009.02.033 (2010).

24. Sudjaroen, Y. et al. Isolation and characterization of ellagitannins as the major polyphenolic components of Longan (Dimocarpus longan Lour) seeds. Phytochem. 77, 226-237 (2012). https://doi.org/ 10.1016/j.phytochem.2011.12.008

25. Zhang, X., Li, N., Lu, X., Liu, P. \& Qiao, X. Effects of temperature on the quality of black garlic. J. Sci. Food Agric. 96 (7), 2366-2372 https://doi.org/10.1002/jsfa.7351 (2016).

26. Abramovič, H., Grobin, B., Poklar Ulrih, N. \& Cigić, B. Relevance and standardization of in vitro antioxidant assays: ABTS, DPPH, and Folin-Ciocalteu. J. Chem. 2018, 4608405. https://doi.org/10.1155/2018/4608405 (2018).

27. Khan, H. et al. Antimalarial and free radical scavenging activities of rhizomes of Polygonatumverticillatum supported by isolated metabolites. Med. Chem. Res. 21 (7), 1278-1282 (2012). https://doi.org/10.1007/ s00044-011-9637-x

28. Marsup, P. et al. Enhancement of Chemical Stability and Dermal Delivery of Cordyceps militaris Extracts by Nanoemulsion. Nanomaterials. 10 (8), 1565 (2020). https:// doi.org/10.3390/nano10081565

29. Nie, S. et al. Effect of $\mathrm{pH}$, temperature and heating time on the formation of furan in sugar-glycine model systems. Food Sci. Hum. Well. 2 (2), 87-92 (2013). https://doi.org/10.1016/j.fshw.2013. 05.001

30. Priyadarsini, K. I., Khopde, S. M., Kumar, S. S. \& Mohan, H. Free radical studies of ellagic acid, a natural phenolic antioxidant. J. Agric. Food Chem. 50 (7), 2200-2206 (2002). https://doi. org/10.1021/jf011275g 
31. Cai, Y. Z., Sun, M., Xing, J., Luo, Q. \& Corke, H. Structure-radical scavenging activity relationships of phenolic compounds from traditional Chinese medicinal plants. Life Sci. 78 (25), 2872-2888 https://doi.org/10.1016/j.lfs.2005.11.004 (2006).

32. Chung, H. Y., Sung, B., Jung, K. J., Zou, Y. \& Yu, B. P. The molecular inflammatory process in aging. Antioxid. Redox. Signal. 8 (3-4), 572-581 https://doi.org/10.1089/ars.2006.8 (2006).

33. Hubbard, R. E., O'Mahony, M. S., Calver, B. L. \& Woodhouse, K. W. Plasma esterases and inflammation in ageing and frailty. Eur. J. Clin. Pharmacol. 64 (9), 895 https://doi.org/10.1007/s00228-008-0499-1 (2008).

34. de Gonzalo-Calvo, D. et al. Differential inflammatory responses in aging and disease: TNF-a and IL-6 as possible biomarkers. Free Radic. Biol. Med. 49 (5), 733-737 https://doi.org/10.1016/j.freeradbiomed.2010.05.019 (2010).

35. Piwowarski, J. P., Kiss, A. K. \& Kozłowska-Wojciechowska, M. Anti-hyaluronidase and anti-elastase activity screening of tannin-rich plant materials used in traditional Polish medicine for external treatment of diseases with inflammatory background. J. Ethnopharmacol. 137 (1), 937-941 https://doi.org/10.1016/j.jep.2011.05.039 (2011).

36. Necas, J., Bartosikova, L., Brauner, P. \& Kolar, J. Hyaluronic acid (hyaluronan): a review. Vet. Med. 53 (8), 397-411 https://doi.org/10.17221/1930-VETMED (2008).

37. Meyer, K. The biological significance of hyaluronic acid and hyaluronidase. Physiol. Rev. 27 (3), 335359 (1947). https://doi.org/ 10.1152/physrev.1947.27.3.335

38. Fam, H., Bryant, J. T. \& Kontopoulou, M. Rheological properties of synovial fluids. Biorheology. 44 (2), 59-74 (2007). https://doi.org/ 10.3233/bir-1987-24404

39. Organización Mundial de la Salud, World Health Organization \& Światowa Organizacja Zdrowia. WHO guidelines on good agricultural and collection practices [GACP] for medicinal plants. World Health Organization, (2003).

40. Chaiyana, W. et al. Ocimum sanctum Linn. as a natural source of skin anti-ageing compounds. Ind. Crops Prod. 127, 217-224 https://doi.org/10.1016/j.indcrop.2018 (2019).

41. Chaiyana, W. et al. Antioxidant, Anti-MMPs, and Anti-Hyaluronidase Activities of Thunbergia laurifolia Lindl. Leaf Extracts for Skin Aging and Skin Damage Prevention. Molecules. 25(8) 1923. https://doi.org/10.3390/ molecules25081923 (2020a).

42. Somwongin, S., Chantawannakul, P. \& Chaiyana, W. Antioxidant activity and irritation property of venoms from Apis species. Toxicon. 145, 32-39 (2018). https://doi.org/10.1016/j.toxicon. 2018.02.049

43. Laothaweerungsawat, N., Neimkhum, W., Anuchapreeda, S., Sirithunyalug, J. \& Chaiyana, W. Transdermal delivery enhancement of carvacrol from Origanum vulgare L. essential oil by microemulsion. Int. J. Pharm. 579, 119052 https://doi.org/10.1016/j.ijpharm.2020 (2020).

44. Mueller, M., Hobiger, S. \& Jungbauer, A. Anti-inflammatory activity of extracts from fruits, herbs and spices. Food Chem. 122 (4), 987-996 https://doi.org/10.1016/j.foodchem.201003.041 (2010). 
45. Chaiyana, W. et al. Enhancement of the Antioxidant, Anti-Tyrosinase, and Anti-Hyaluronidase Activity of Morus alba L. Leaf Extract by Pulsed Electric Field Extraction. Molecules. 25 (9), 2212 https://doi.org/10.3390/molecules25092212 (2020b).

\section{Figures}

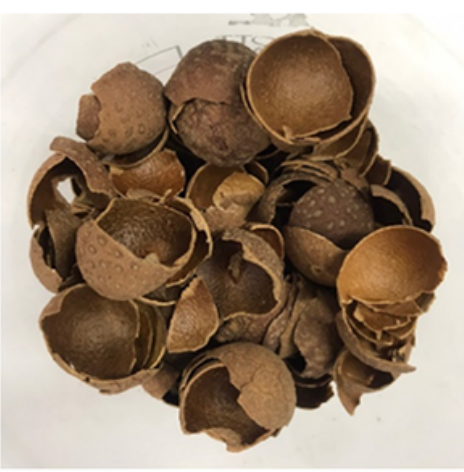

(a)

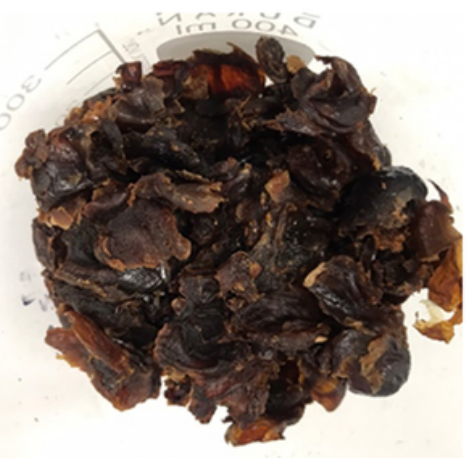

(e)

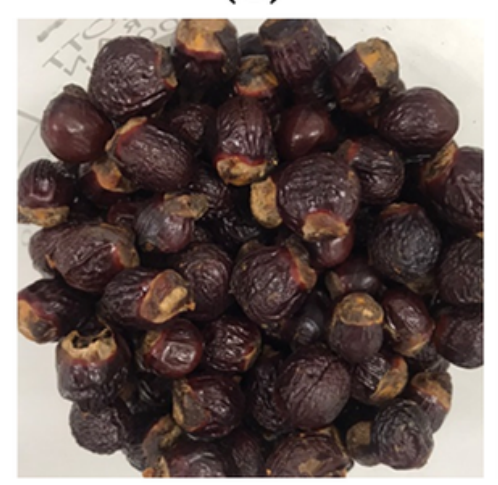

(i)

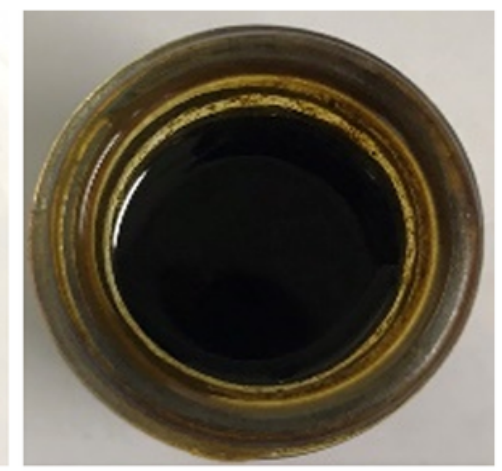

(b)

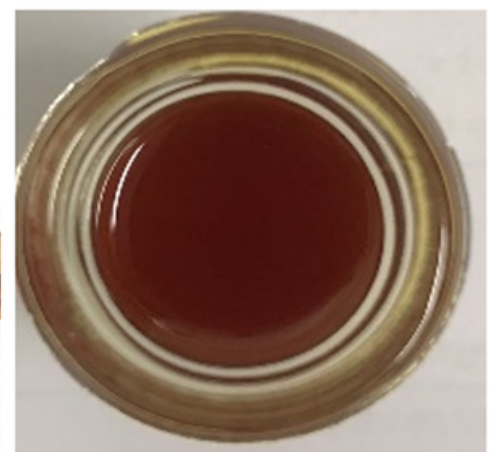

(f)

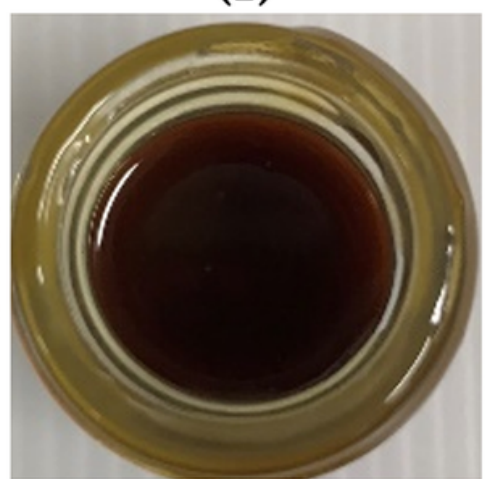

(j)

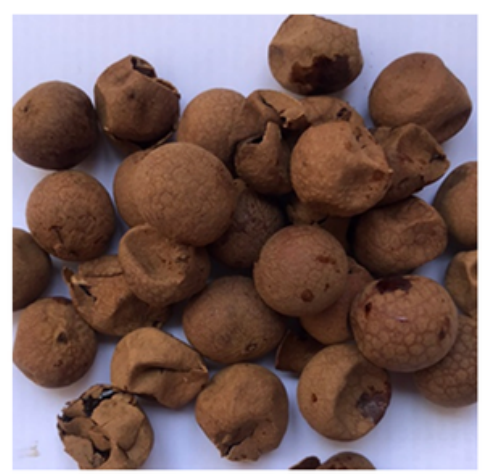

(c)

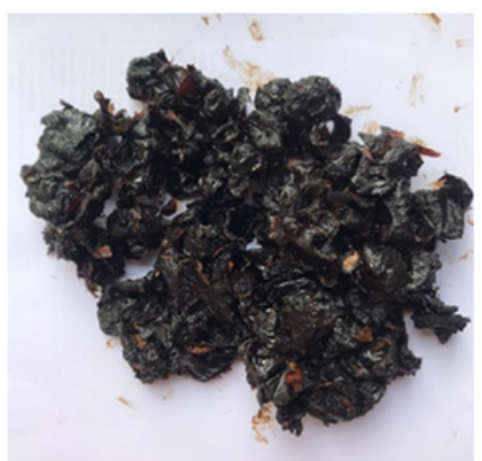

(g)

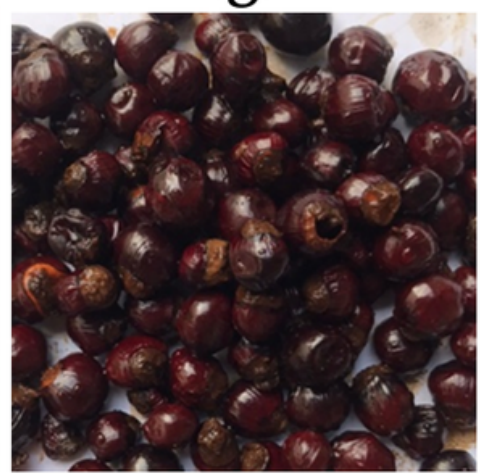

(k)

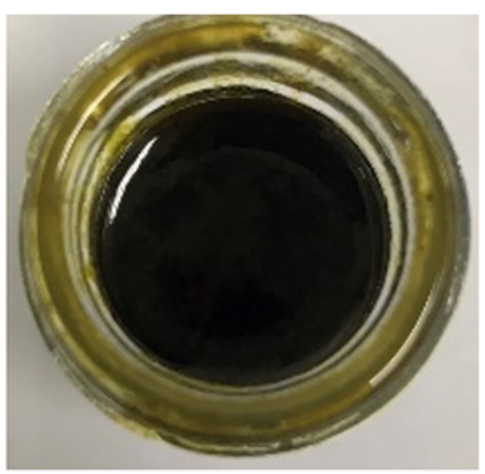

(d)

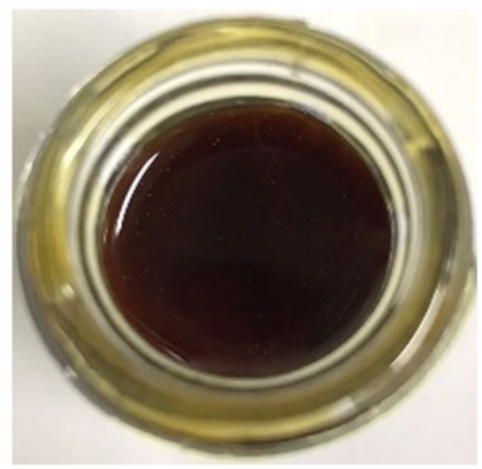

(h)

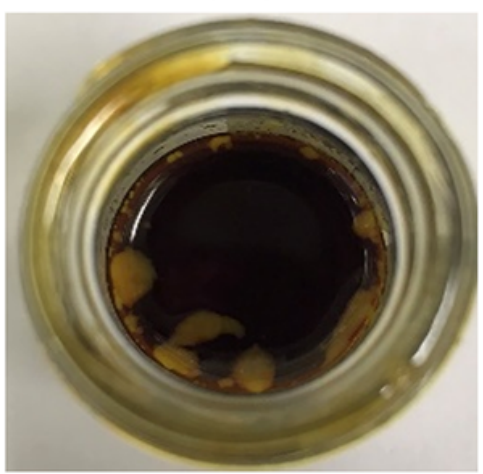

(1)

\section{Figure 1}

External appearance of dried D. longan pericarp (a), dried D. longan pericarp extract (b), black D. longan pericarp (c), black D. longan pericarp extract (d), dried D. longan aril (e), dried D. longan aril extract (f), black $D$. longan aril $(g)$, black $D$. longan aril extract $(h)$, dried $D$. longan seed (i), dried D. longan seed extract $(\mathrm{j})$, black $\mathrm{D}$. longan seed $(\mathrm{k})$, black $\mathrm{D}$. longan seed extract (I). 


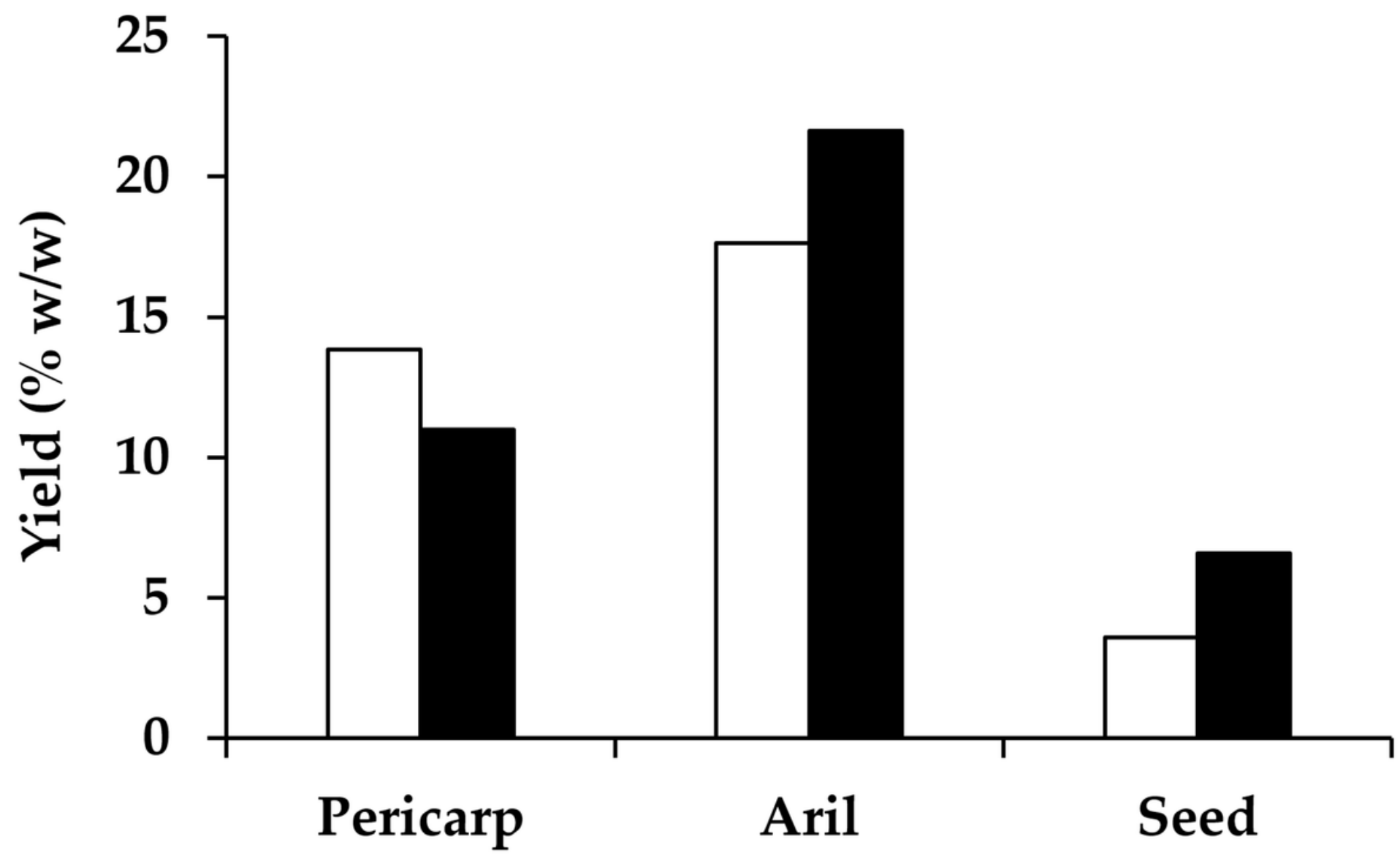

Figure 2

Yield of the ethanolic extracts from pericarp, aril, and seed part of dried ( $\square$ ) and black ( $\square$ ) D. longan. 
(a)

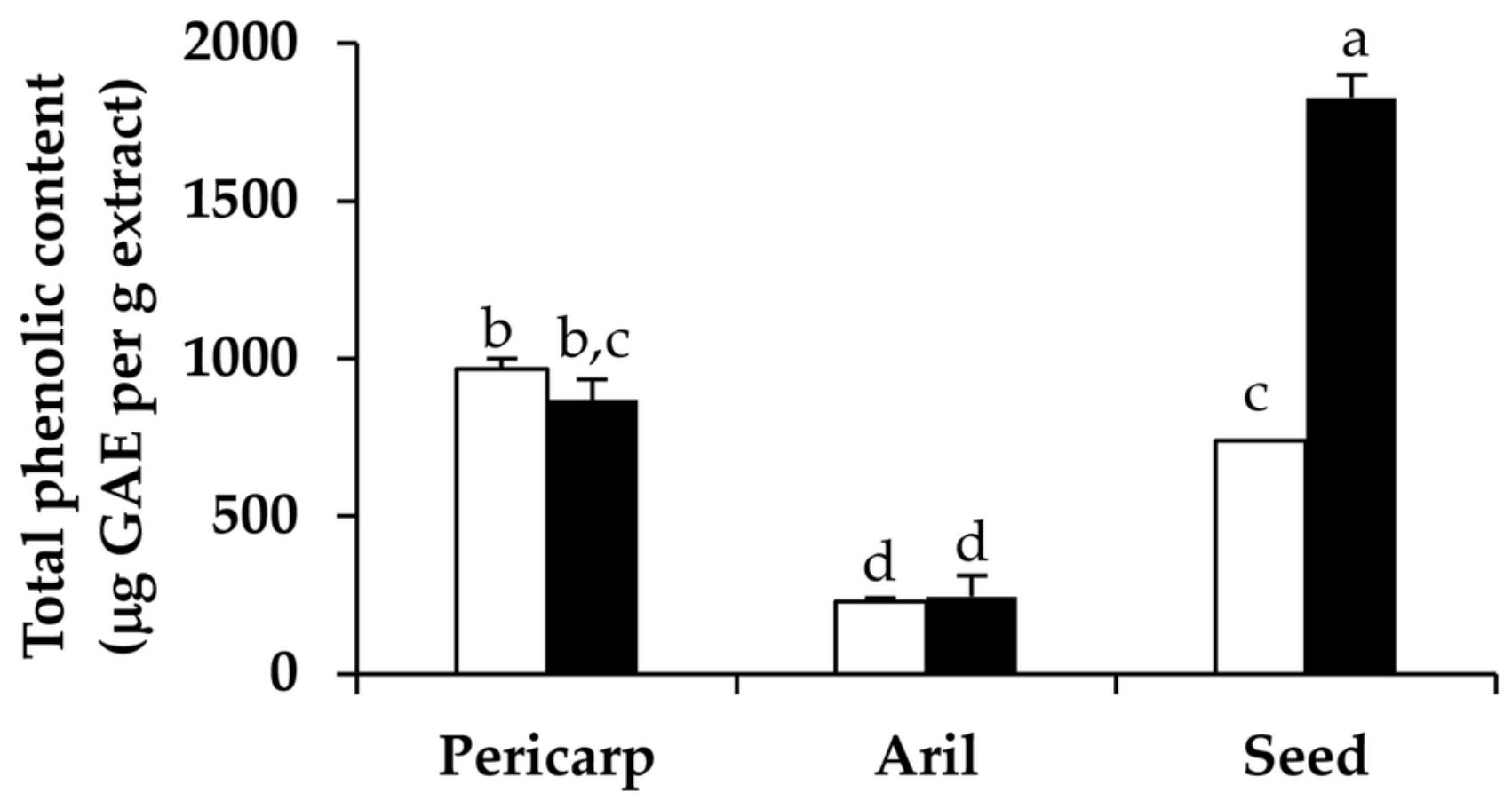

(b)

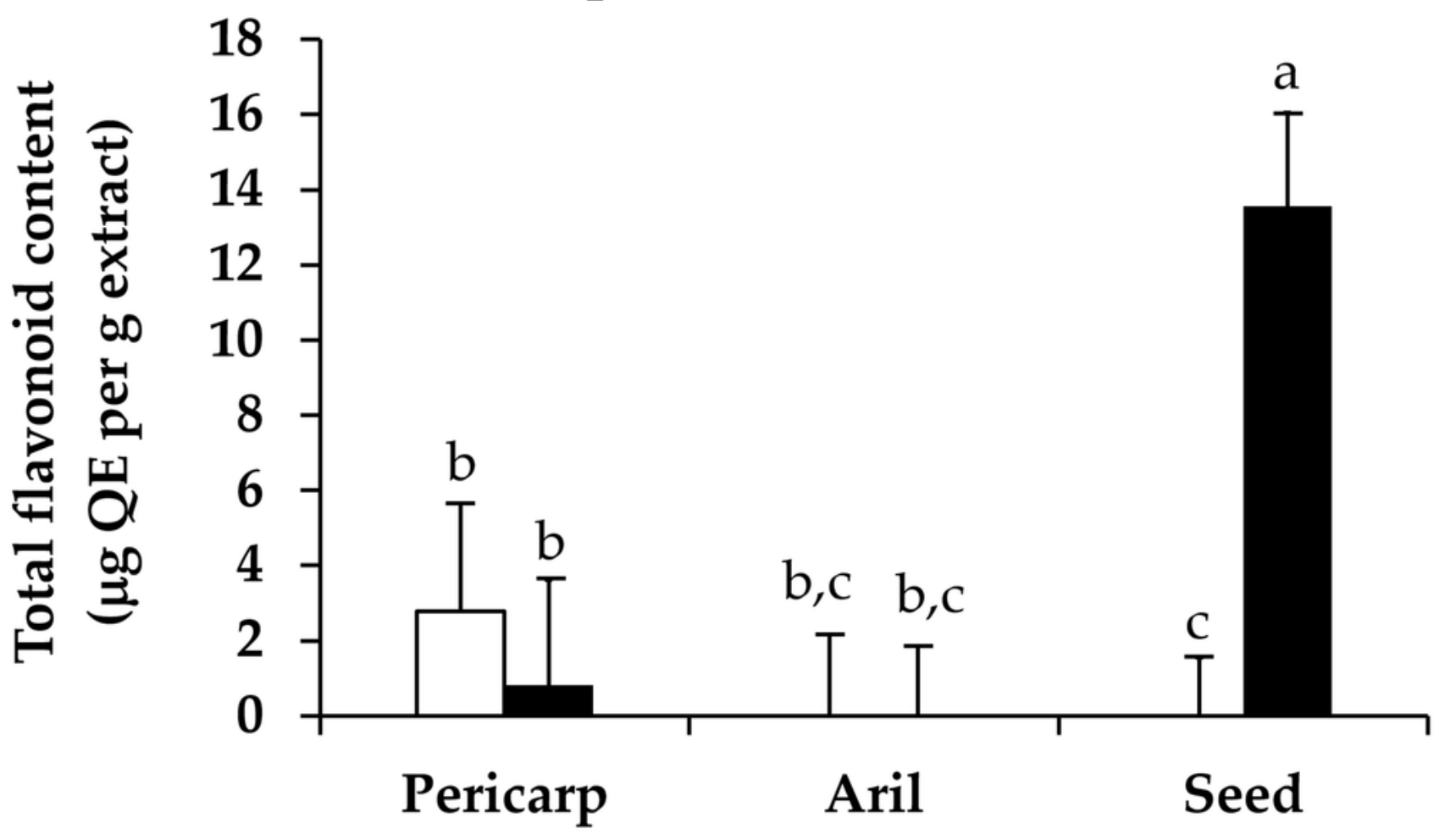

Figure 3

Total phenolic content (A) and total flavonoid content (B) of the ethanolic extracts from pericarp, aril, and seed part of dried ( $\square$ ) and black ( $\square$ ) D. longan. The letters (a, b, c, and d) denote significantly differences in total phenolic content or total flavonoid content among various $D$. longan extracts $(p<0.05)$. 

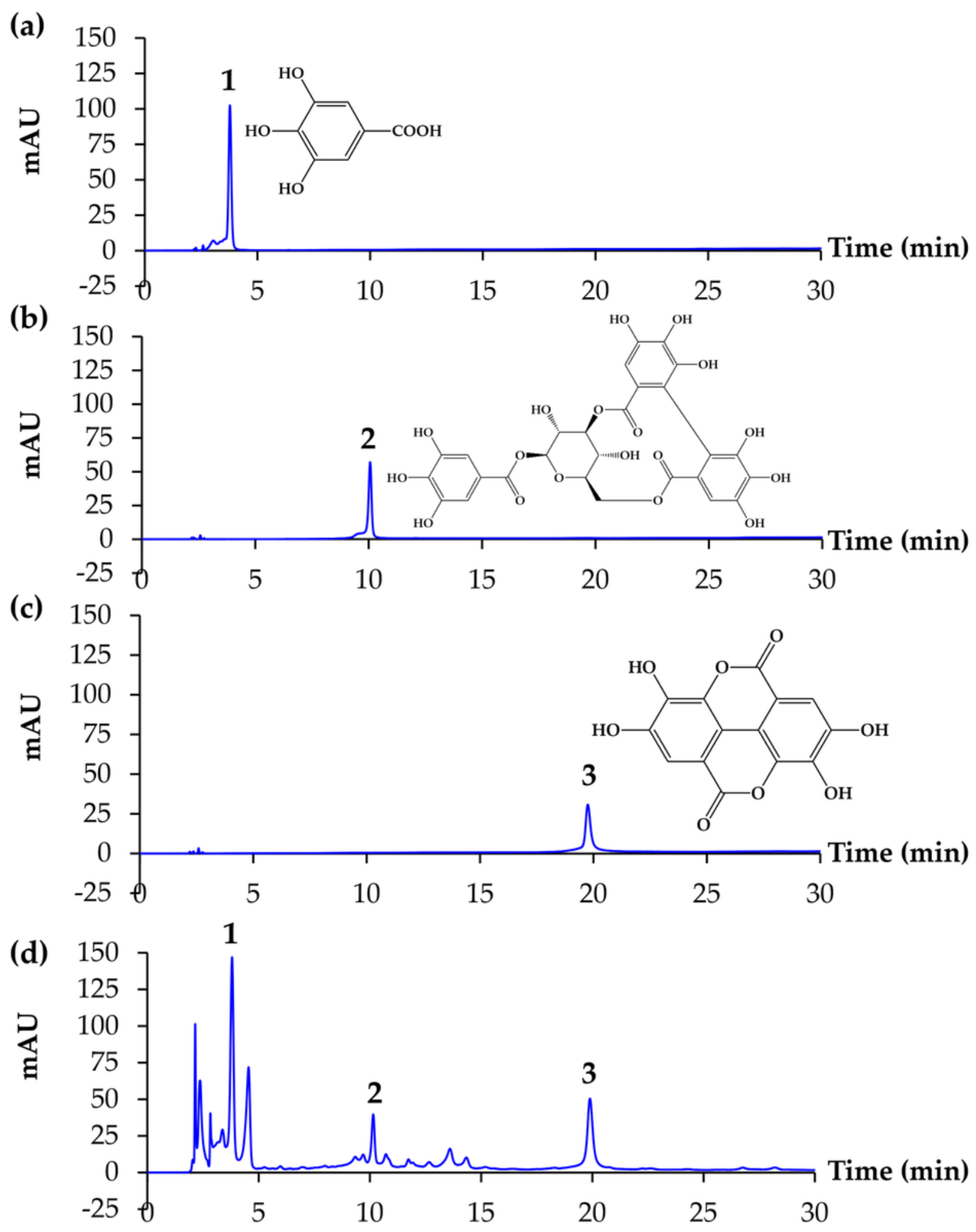

Figure 4

HPLC chromatograms of gallic acid (a), corilagin (b), ellagic acid (c), and black D. longan seed extract (d). 
(a)
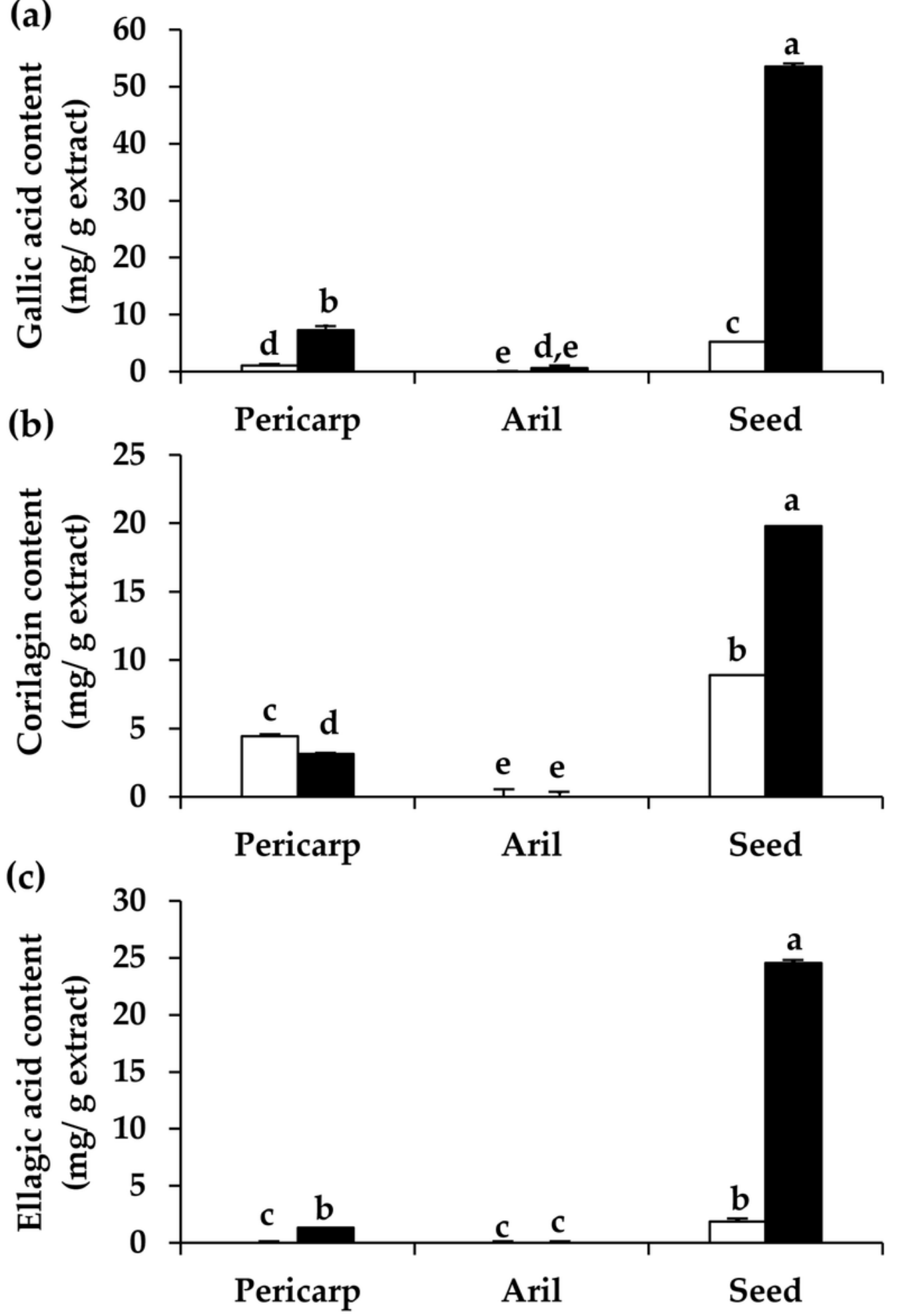

Figure 5

Gallic acid content (a), corilagin content (b), and ellagic acid content (c) of the ethanolic extracts from pericarp, aril, and seed part of dried ( $\square$ ) and black ( $\square$ ) D. longan. The letters (a, b, c, d, and e) denote significantly differences in the content of gallic acid, corilagin, or ellagic acid among various $\mathrm{D}$. longan extracts $(p<0.05)$. 

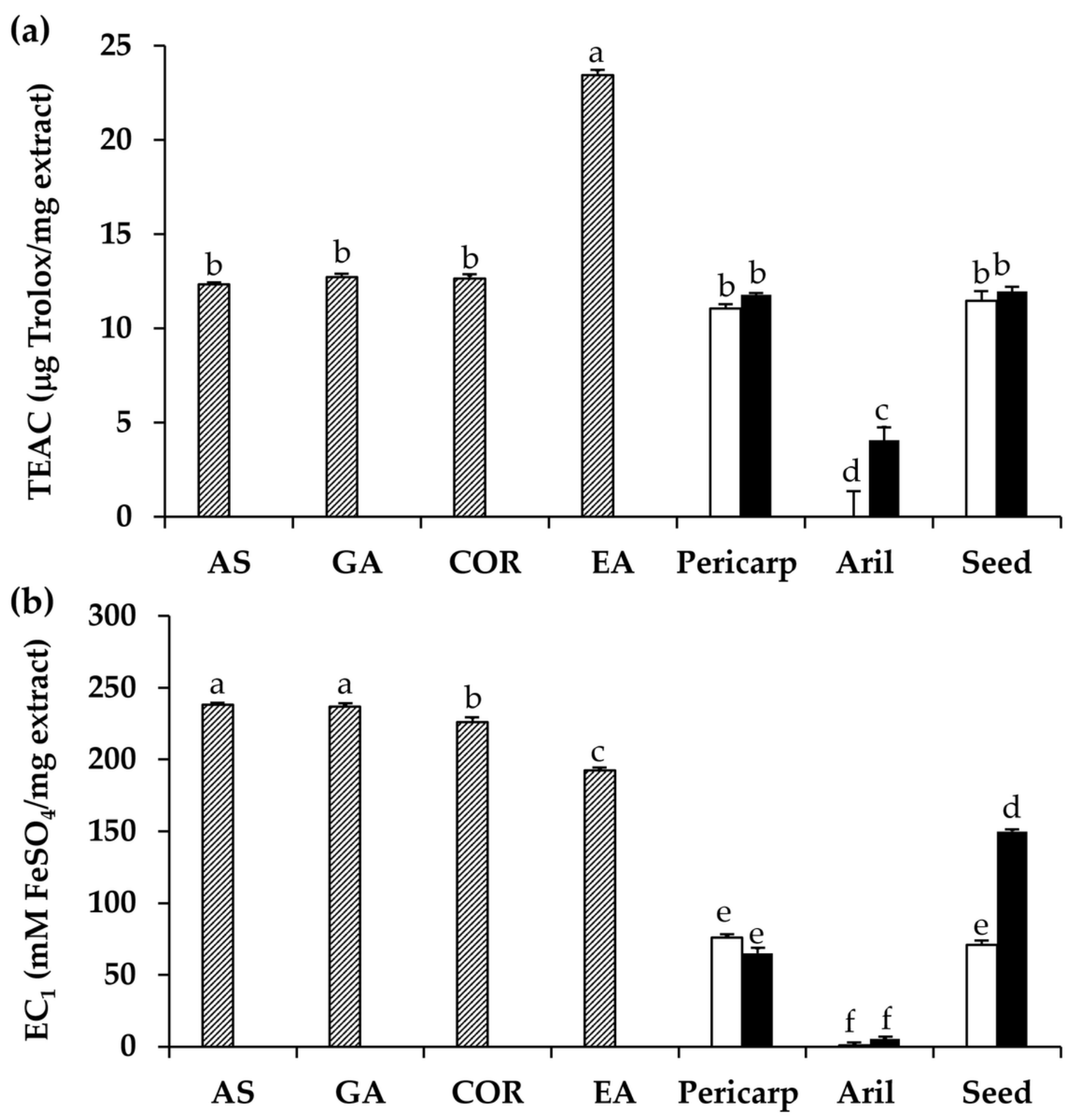

Figure 6

Trolox equivalent antioxidant capacity (TEAC) (a) and equivalent concentration (EC1) (b) of ascorbic acid (AS), gallic acid (GA), corilagin (CO), ellagic acid (EA), and the ethanolic extracts from pericarp, aril, and seed part of dried ( $\square$ ) and black ( $\square$ ) D. longan. The letters (a, b, c, d, e, and f) denote significantly differences in TEAC or EC1 values among various tested samples $(p<0.05)$. 

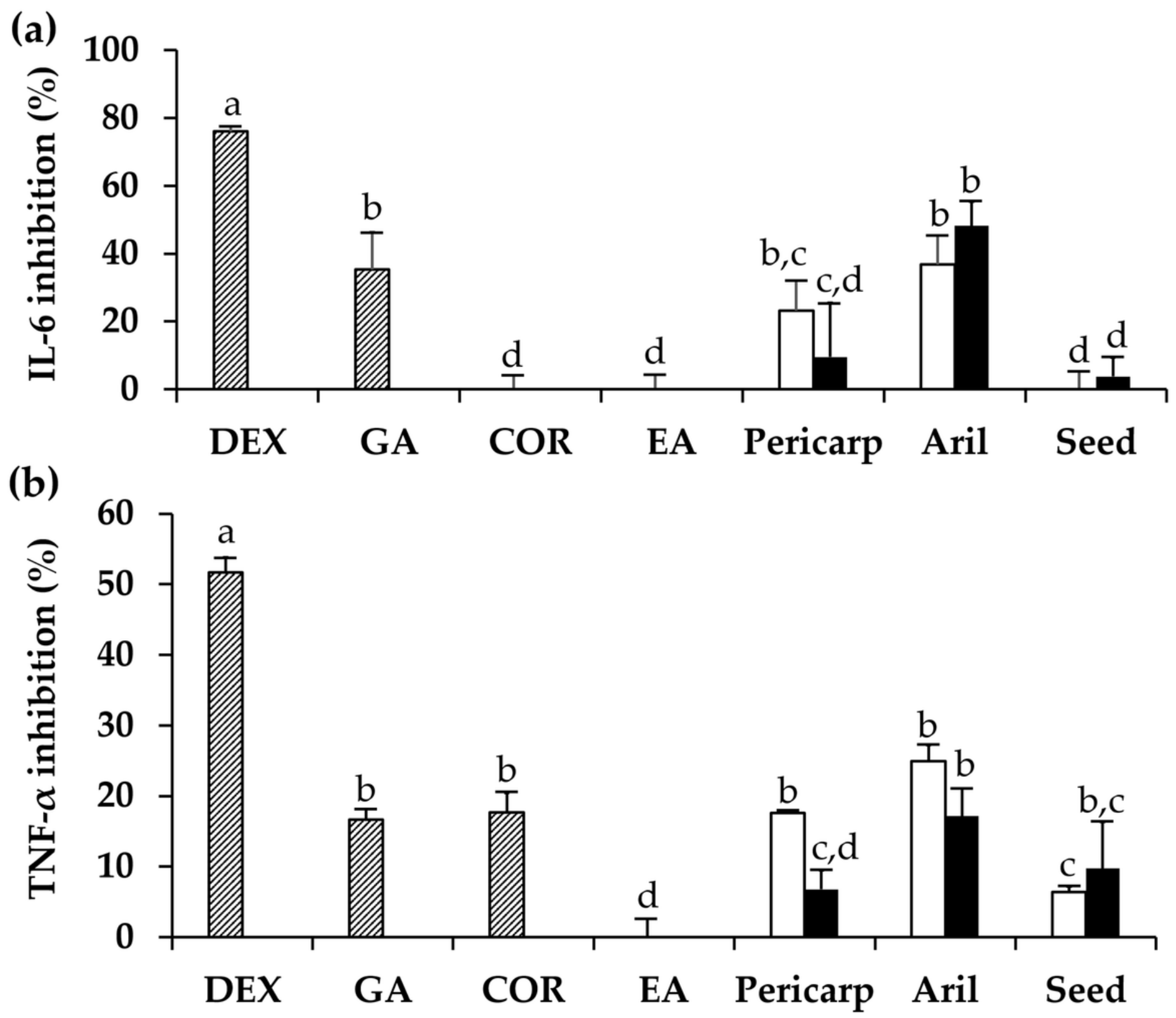

Figure 7

Inhibitory activities against the secretion of interleukin-6 (IL-6) (a) and tumor necrosis factor-a (TNF- a) of dexamethasone (DEX), gallic acid (GA), corilagin (CO), ellagic acid (EA), and the ethanolic extracts from pericarp, aril, and seed part of dried $(\square)$ and black ( $\square$ ) D. longan. The letters (a, b, c, and d) denote significantly differences in IL-6 or TNF- $a$ inhibition among various tested samples $(p<0.05)$. 


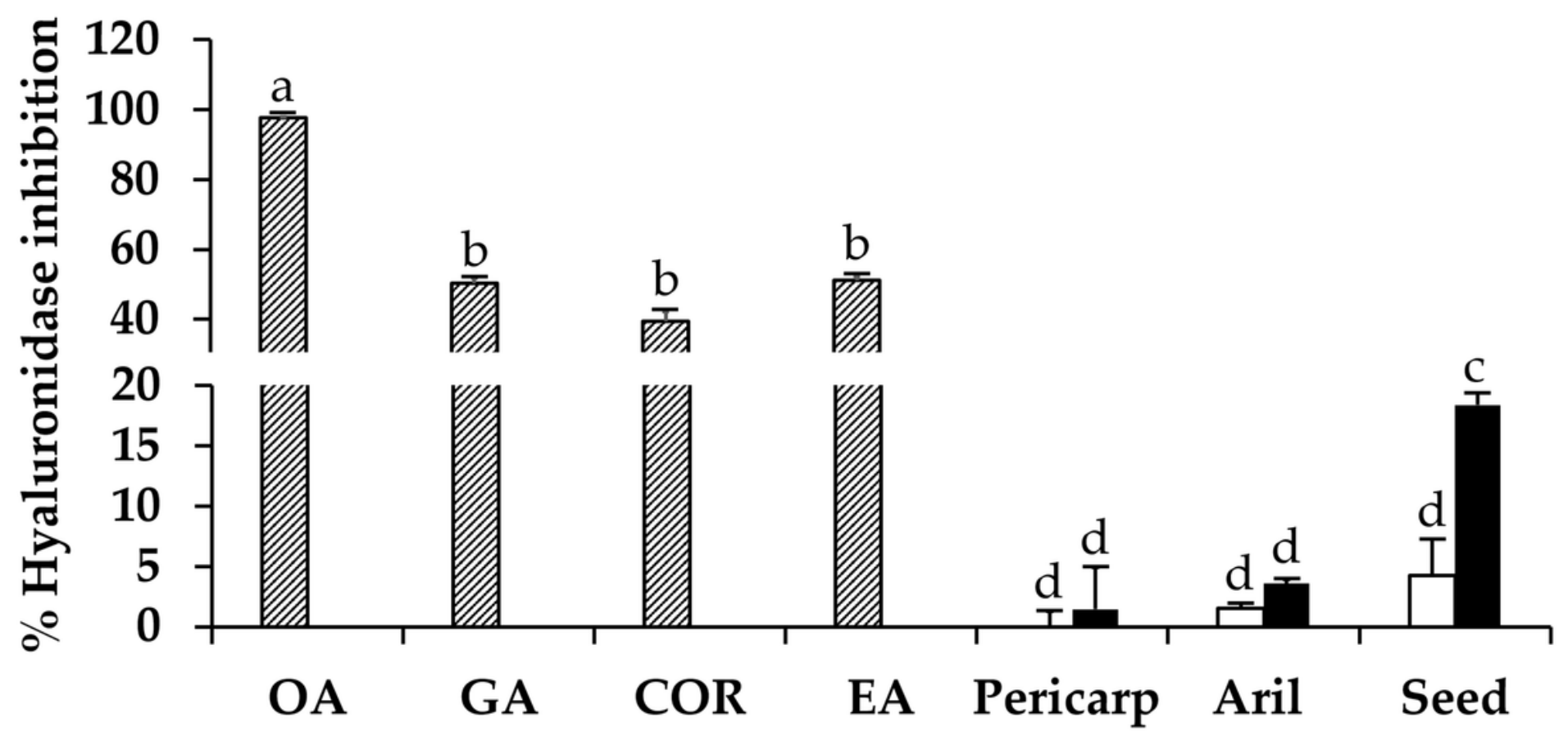

Figure 8

Inhibitory activities against hyaluronidase activity of oleanolic acid (OA), gallic acid (GA), corilagin (CO), ellagic acid (EA), and the ethanolic extracts from pericarp, aril, and seed part of dried ( $\square$ ) and black ( $\square$ ) D. longan. The letters $(a, b, c$, and $d)$ denote significantly differences in hyaluronidase inhibition among various tested samples $(p<0.05)$. 